\title{
Continuous regional arterial infusion for acute pancreatitis: a propensity score analysis using a nationwide administrative database
}

Tsuyoshi Hamada', Hideo Yasunaga ${ }^{2}$, Yousuke Nakai ${ }^{1}$, Hiroyuki Isayama ${ }^{*}$, Hiromasa Horiguchi ${ }^{3}$, Shinya Matsuda ${ }^{4}$, Kiyohide Fushimi ${ }^{5}$ and Kazuhiko Koike

\begin{abstract}
Introduction: Although continuous regional arterial infusion (CRAl) of a protease inhibitor and an antibiotic may be effective in patients with severe acute pancreatitis, CRAl has not yet been validated in large patient populations. We therefore evaluated the effectiveness of CRAI based on data from a national administrative database covering 1,032 Japanese hospitals.
\end{abstract}

Methods: In-hospital mortality, length of stay and costs were compared in the CRAl and non-CRAl groups, using propensity score analysis to adjust for treatment selection bias.

Results: A total of 17,415 eligible patients with acute pancreatitis were identified between 1 July and 30 September 2011, including 287 (1.6\%) patients who underwent CRAl. One-to-one propensity-score matching generated 207 pairs with well-balanced baseline characteristics. In-hospital mortality rates were similar in the CRAI and non-CRAI groups (7.7\% vs. 8.7\%; odds ratio, $0.88 ; 95 \%$ confidence interval, $0.44-1.78, P=0.720$ ). CRAI was associated with significantly longer median hospital stay ( 29 vs. 18 days, $P<0.001$ ), significantly higher median total cost $(21,800$ vs. 12,600 United States dollars, $P<0.001$ ), and a higher rate of interventions for infectious complications, such as endoscopic/surgical necrosectomy or percutaneous drainage ( $2.9 \%$ vs. $0.5 \%, P=0.061)$.

Conclusions: CRAI was not effective in reducing in-hospital mortality rate in patients with acute pancreatitis, but was associated with longer hospital stay and higher costs. Randomized controlled trials in large numbers of patients are required to further evaluate CRAI for this indication.

\section{Introduction}

Acute pancreatitis is an inflammatory disease of the pancreas with a wide spectrum of severity. It is characterized by autodigestion of the pancreas due to activation of inherent proteases and frequently involves surrounding retroperitoneal tissues and/or remote organ systems. Although recent advances in intensive care and aggressive treatment methods specialized for acute pancreatitis $[1,2]$ have dramatically reduced the mortality rate associated with this condition, mortality due to severe necrotizing pancreatitis is not uncommon [3,4]. Protease inhibitors may directly suppress pancreatic inflammation

\footnotetext{
* Correspondence: isayama-tky@umin.ac.jp

'Department of Gastroenterology, Graduate School of Medicine, The University of Tokyo, 7-3-1 Hongo, Bunkyo-ku, Tokyo 113-8655, Japan Full list of author information is available at the end of the article
}

by inhibiting pancreatic enzymes and improving coagulopathy $[5,6]$. In addition, antibiotics have been reported to reduce the mortality associated with severe acute pancreatitis by preventing secondary infection of the necrotic pancreatic tissue $[7,8]$, although its effectiveness on mild acute pancreatitis has not been demonstrated [3]. In acute necrotizing pancreatitis, however, pancreatic ischemia due to vasospasm and enhanced intravascular coagulation inhibit protease inhibitors and prevent intravenously administered antibiotics from penetrating into pancreatic tissue.

Continuous regional arterial infusion (CRAI), rather than systemic intravenous administration, may be a theoretically reasonable drug delivery system for patients with severe acute pancreatitis. Administration of a protease inhibitor through a catheter placed into one of the arteries supplying the inflamed pancreas can dramatically increase the effective concentration of this agent in 
the pancreatic parenchyma $[9,10]$. To date, several retrospective, uncontrolled case series have suggested that CRAI may reduce mortality associated with acute necrotizing pancreatitis [11-15]. To the best of our knowledge, however, only one randomized controlled trial has assessed mortality rates in patients administered a protease inhibitor and an antibiotic via CRAI [16]. In that study, which involved 78 patients with severe acute pancreatitis, the mortality rate was significantly lower in the CRAI group than in the non-CRAI group (5.1\% vs. $23.1 \%$; $P=0.02$ ). Owing to the small sample size and lack of stratification, however, the characteristics of patients in the CRAI and non-CRAI groups were poorly balanced, with the CRAI group being younger and having a better computed tomography $(\mathrm{CT})$ severity index, possibly leading to an overestimation of the efficacy of CRAI [17]. Thus, the effectiveness of CRAI for acute necrotizing pancreatitis remains unclear.

We therefore evaluated the effectiveness of CRAI of a protease inhibitor and an antibiotic in patients with acute pancreatitis by performing a one-to-one propensity score-matched analysis of patients with or without CRAI derived from a nationwide administrative database of inpatient care in Japan.

\section{Materials and methods}

\section{Data source}

The Diagnosis Procedure Combination (DPC) database is an original case mix administrative database of inpatients in Japan that provides data on admission and discharge abstracts as well as administrative claims. The DPC system has been adopted by acute care hospitals in Japan $[18,19]$. Data on approximately seven million inpatients hospitalized between 1 July 2010 and 30 June 2011 were collected from more than 1,000 hospitals throughout Japan, representing approximately $50 \%$ of all acute care hospitalizations during the same period in Japan. The main diagnoses, comorbidities present at admission and complications during hospitalization were recorded using the International Classification of Diseases and Related Health Problems, 10th Revision (ICD-10) codes and text data in the Japanese language. The database also contains detailed medical information, such as patients' age and sex; length of hospital stay; discharge status, including in-hospital death; unique identifiers of the hospitals; types of hospitals (academic and nonacademic); interventional and surgical procedures; medications and devices indexed by the original codes in Japanese; and cost data. The database also includes consciousness status at the time of admission and discharge based on the Japan Coma Scale (JCS), in which a score of 0 indicates alert consciousness, scores of 1 to 3 indicate wakefulness without any stimuli, scores of 10 to 30 indicate arousal by some stimuli and scores of 100 to 300 indicate coma. The JCS and the Glasgow Coma Scale assessments are well-correlated [20,21]. Each patient with a principal diagnosis of acute pancreatitis was given the highest prognostic factor and CT severity index scores within 48 hours of admission according to the Japanese severity scoring system by the attending physicians [22,23].

This study was approved by the institutional review board of The University of Tokyo Hospital, which waived the requirement for patient informed consent because of the anonymous nature of the data.

\section{Japanese severity scoring system for acute pancreatitis}

The severity of acute pancreatitis was determined for each patient on the basis of the Japanese severity score (prognostic factor score) determined by summing nine factors, along with the CT severity score [22,24]. Table 1 shows the details of this scoring system. Severe acute pancreatitis was diagnosed when the total prognostic factor score was 3 or higher or the CT severity grade was 2 or higher [22].

\section{Continuous regional arterial infusion of a protease inhibitor and an antibiotic}

CRAI consisted of the continuous infusion of a protease inhibitor and an antibiotic (usually carbapenem) through a catheter inserted into one of the arteries perfusing the inflamed lesion of the pancreas. The general methods of CRAI were as follows. The catheter used for CRAI was the same as the one used for angiography. Following CT evaluation of the hypoenhanced area of the pancreas, angiography of the pancreas was performed. The catheter tip was located in the artery perfusing the area containing the main lesion of hypoperfusion of the pancreas. If the main lesion was located in the head of the pancreas, the catheter tip was placed in the common hepatic, gastroduodenal or superior mesenteric artery; if the main lesion was located in the body or tail of the pancreas, the catheter tip was placed in the celiac, splenic or dorsal pancreatic artery; and if the lesion involved the entire pancreas, the catheter tip was placed in the celiac artery. CRAI was usually started within 2 or 3 days of admission.

\section{Patient selection and data}

We identified all adult patients (20 years of age or older) who were admitted to the participating hospitals with a principal diagnosis of acute pancreatitis (ICD-10 code K85) and were discharged between 1 July 2010 and 30 September 2011. Patients transferred within 7 days of hospitalization were excluded because transfer was based on Japanese guidelines recommending that patients with a prognostic factor score of 3 or higher be transferred to a specialized medical institution [22]. Patients who underwent CRAI for acute pancreatitis were identified based on the performance of selective arterial angiography combined with the infusion of a protease inhibitor (gabexate mesilate or nafamostat mesilate) and a 
Table 1 Japanese severity scoring system for acute pancreatitis of the Ministry of Health, Labour and Welfare of Japan (2008 revision) ${ }^{a}$

\begin{tabular}{|c|c|}
\hline Scoring system & Basis for score \\
\hline \multicolumn{2}{|c|}{$\begin{array}{l}\text { Prognostic factor score (one point for } \\
\text { each factor) }\end{array}$} \\
\hline 1 & Base excess less than or equal to $-3 \mathrm{mEq} / \mathrm{L}$ or shock (systolic blood pressure below $80 \mathrm{mmHg}$ ) \\
\hline 2 & $\mathrm{PaO}_{2} \leq 60 \mathrm{mmHg}$ (room air) or respiratory failure (respiratory assistance needed) \\
\hline 3 & $\begin{array}{l}\text { BUN } \geq 40 \mathrm{mg} / \mathrm{dl} \text { or (or creatinine } \geq 2.0 \mathrm{mg} / \mathrm{dl} \text { ) or oliguria (daily urine output }<400 \mathrm{ml} \text { even after } \\
\text { intravenous fluid resuscitation) }\end{array}$ \\
\hline 4 & LDH at or above twice the upper limit of normal \\
\hline 5 & Platelet count $\leq 100,000 / \mathrm{mm}^{3}$ \\
\hline 6 & Serum calcium $\leq 7.5 \mathrm{mg} / \mathrm{dl}$ \\
\hline 7 & $\mathrm{CRP} \geq 15 \mathrm{mg} / \mathrm{dl}$ \\
\hline 8 & Number of positive measures in SIRS criteria $\geq 3$ \\
\hline 9 & Age $\geq 70$ years \\
\hline \multicolumn{2}{|c|}{$C T$ grade based on contrast-enhanced $C T$} \\
\hline \multirow[t]{4}{*}{1} & Extrapancreatic progression of inflammation \\
\hline & Anterior pararenal space, zero points \\
\hline & Root of mesocolon, one point \\
\hline & Beyond lower pole of kidney, two points \\
\hline \multirow[t]{9}{*}{2} & Hypoenhanced lesion of the pancreas \\
\hline & Pancreas conveniently divided into three segments (head, body and tail). \\
\hline & Localized in each segment or surrounding only the pancreas, zero points \\
\hline & Extends to two segments, one point \\
\hline & Occupies two whole segments or more, two points \\
\hline & Factors $1+2=$ total score \\
\hline & Total score $=0$ or 1 , grade 1 \\
\hline & Total score $=2$, grade 2 \\
\hline & Total score $=3$ or more, grade 3 \\
\hline Assessment of severity & If prognostic factor score is $\geq 3$ or $C T$ grade is $\geq 2$, acute pancreatitis is considered severe. \\
\hline
\end{tabular}

${ }^{\mathrm{a}} \mathrm{BUN}$, Blood urea nitrogen; CRP, C-reactive protein; CT, Computed tomography; $\mathrm{LDH}$, Lactate dehydrogenase; $\mathrm{PaO}_{2}$, partial pressure of oxygen in blood; SIRS, Systemic inflammatory response syndrome. Measures within the SIRS criteria include body temperature above $38^{\circ} \mathrm{C}$ or less than $36^{\circ} \mathrm{C}$, heart rate more than 90 beats/min, respiratory rate more than 20 breaths/min or partial pressure of carbon dioxide in blood less than 32 torr, as well as white blood cell count above 12,000 cells $/ \mathrm{mm}^{3}$, less than 4,000 cells $/ \mathrm{mm}^{3}$ or greater than $10 \%$ immature (band) forms. The 2008 revision of the Japanese severity scoring system is contained in [22].

carbapenem antibiotic (meropenem, imipenem, doripenem, biapenem or panipenem). The cohort for propensity score analysis was generated by combining those patients who underwent CRAI within 3 days of admission and patients who had intravenous infusions with both a protease inhibitor and a carbapenem antibiotic within 3 days of admission. The types of protease inhibitors and antibiotics initially administered via CRAI were also recorded.

The baseline characteristics, prognostic factor scores and CT severity scores at the time of admission of each patient were recorded. JCS $[20,21]$ was categorized into four groups: 0,1 to 3,10 to 30 and 100 to 300 . Comorbidities, assessed by recording ICD-10 codes, were converted into scores, and these scores were summed to calculate the Charlson comorbidity index (CCI) score for each patient based on Quan's algorithm [25]. Hospital volume was defined as the number of inpatients with acute pancreatitis per year per hospital and was categorized into quartiles (very low, low, high and very high volume) containing approximately equal numbers of patients [26]. Hospital type was categorized as academic or nonacademic.

\section{Outcomes}

The primary outcome of this study was in-hospital mortality. Secondary outcomes included length of hospital stay, total costs and requirement of intervention for infectious complications, that is, endoscopic or surgical necrosectomy or percutaneous drainage.

\section{Statistical analyses}

A retrospective observational design was utilized to evaluate the effectiveness of CRAI of a protease inhibitor 
and an antibiotic for acute pancreatitis. Because CRAI is likely to be performed in more severely ill patients, an unadjusted comparison of CRAI and non-CRAI groups may be subject to treatment selection bias $[27,28]$, resulting in an underestimation of the effectiveness of CRAI. To overcome this bias, we performed a propensity score analysis $[27,28]$. A propensity score was calculated for each patient in the cohort as described, using a logistic regression model with baseline variables that potentially influenced the selection of CRAI, including age, sex, JCS score, CCI score, prognostic factor score, CT severity index score, hospital volume and hospital type. We then matched patients in the CRAI patient cohort one-to-one with patients in the non-CRAI group by propensity score matching using the estimated propensity scores of each patient based on the nearest neighbor method within a caliper. In this algorithm, each patient in the non-CRAI group was matched with a patient in the CRAI group with the closest estimated propensity score within a specified range $(\leq 0.25$ of the pooled standard deviation of estimated propensity scores) [29]. C-statistics were used to evaluate goodness of fit.

Continuous variables were described as means and standard deviations or medians and interquartile ranges (IQRs) and were compared using Student's $t$-test or the Mann-Whitney $U$ test as appropriate. Between-group differences in categorical variables were compared using Fisher's exact test or a $\chi^{2}$ test as appropriate. The trend toward higher in-hospital mortality with later administration of CRAI was evaluated using the CochraneArmitage trend test. A multivariate logistic regression model was used to compare in-hospital mortality in the CRAI and non-CRAI groups, with adjustment of propensity score quintiles.

The Cochrane-Armitage trend test was performed using $\mathrm{R}$ software version 2.15.1 ( $\mathrm{R}$ Development Core Team; http://www.r-project.org). All other statistical analyses were performed using IBM SPSS Statistics version 19 software (IBM, Armonk, NY, USA). $P<0.05$ was considered statistically significant.

\section{Results}

\section{Patient selection and matching}

During the 15-month study period, 21,468 patients ages 20 years and older were hospitalized with acute pancreatitis at the 1,032 DPC participating hospitals (116 academic and 916 nonacademic hospitals). We identified 17,415 patients whose prognostic factor scores and CT severity scores upon admission were both recorded and who had not been transferred within 7 days of hospitalization. From among these 17,415 patients, 287 (1.6\%) underwent CRAI at 29 academic and 70 nonacademic hospitals. The cohort used for propensity score analysis consisted of 247 patients who underwent CRAI within 3 days of admission (the CRAI group) and 1,307 patients who underwent intravenous administration of a protease inhibitor and a carbapenem antibiotic within 3 days of admission (the non-CRAI group).

Patients in the CRAI and non-CRAI groups had mean propensity scores of 0.334 (95\% confidence interval $(\mathrm{CI})=0.308$ to 0.360$)$ and $0.126(95 \% \mathrm{CI}=0.119$ to 0.133$)$, respectively. The $\mathrm{C}$-statistic (area under the receiver operating characteristic curve) was 0.811 . Using the algorithm described above, we were able to match 207 patients in the CRAI group with 207 in the non-CRAI group (Figure 1).

\section{Patient characteristics}

The characteristics of all patients in the CRAI and nonCRAI groups $(n=1,554)$ and in the 207 propensitymatched pairs $(n=414)$ are shown in Table 2. Analysis of the total patient population showed that patients in the CRAI group was younger and more likely to be treated in academic hospitals, and they had higher prognostic factor and CT severity index scores. CRAI was administered on day 1 of admission to 109 patients (52.7\%), on day 2 to 60 patients $(29.0 \%)$, on day 3 to 31 patients (15.0\%) and on day 4 to 7 patients (3.4\%).

Propensity score matching revealed that patient characteristics were similar in the adjusted CRAI and nonCRAI groups. In particular, the CT severity scores did not differ significantly between groups.

The protease inhibitors gabexate mesilate and nafamostat mesilate were administered to $60.4 \%$ and $39.6 \%$, respectively, of the patients in the propensity-matched CRAI group, and to $58.9 \%$ and $41.1 \%$, respectively, of the patients in the propensity-matched non-CRAI group. The antibiotics meropenem, imipenem and others were administered to $44.0 \%, 43.0 \%$ and $13.0 \%$, respectively, of the patients in the propensity-matched CRAI group, and to $60.9 \%, 32.9 \%$ and $6.2 \%$, respectively, of the patients in the propensity-matched non-CRAI group.

\section{Outcomes in the CRAI and non-CRAI groups}

Among all 1,554 eligible patients, the in-hospital mortality rate was higher in the CRAI group than in the nonCRAI group ( $8.5 \%$ vs. $6.8 \% ; P=0.342)$. Outcomes in the propensity-matched CRAI and non-CRAI groups are summarized in Table 3. In-hospital mortality rates were similar (7.7\% vs. $8.7 \%$, odds ratio $(\mathrm{OR})=0.88,95 \% \mathrm{CI}=0.44$ to 1.78; $P=0.720)$. In-hospital mortality rates were $4.6 \%$ in patients who underwent CRAI on admission day 1 , $10.0 \%$ on day 2 and $13.2 \%$ on day 3 or later. Earlier administration of CRAI tended to be associated with lower in-hospital morality rate $(P=0.064)$. In addition, length of hospital stay was significantly longer $(P<0.001)$ and total costs during hospitalization were significantly higher $(P<0.001)$ in the matched CRAI group than in 


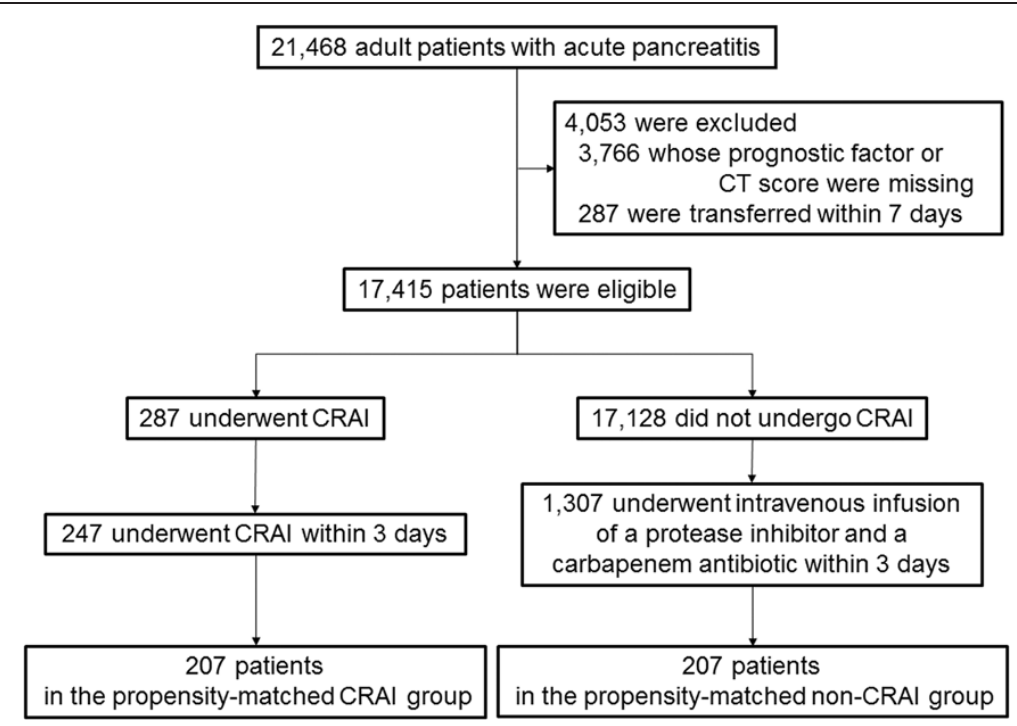

Figure 1 Flowchart of patients with acute pancreatitis and propensity matching of patients with or without continuous regional arterial infusion. CRAI, Continuous regional arterial infusion; CT, Computed tomography.

the matched non-CRAI group, whereas the rate of interventions for infectious complications tended to be higher in the matched CRAI group $(2.9 \%$ vs. $0.5 \% ; P=0.061)$. In detail, four patients in the matched CRAI group and one in the matched non-CRAI group required endoscopic necrosectomy, and two patients in the matched CRAI group required percutaneous drainage. No patient required surgical necrosectomy.

Logistic regression analysis with adjustment for propensity score quintiles showed that the in-hospital mortality rate (adjusted $\mathrm{OR}=0.88 ; 95 \% \mathrm{CI}=0.43$ to $1.78 ; P=0.711)$ and the rate of interventions for infectious complications (adjusted $\mathrm{OR}=6.42,95 \% \mathrm{CI}=0.75$ to $54.6 ; P=0.089$ ) were similar in the matched CRAI and non-CRAI groups (Table 4). Linear regression analysis with adjustment for the same variables as those used in the calculation of propensity scores showed that hospital stay was 16.5 days longer $(P<0.001)$ and cost was US $\$ 13,600$ higher $(P<0.001)$ in the matched CRAI cohort than in the matched non-CRAI group (Table 4).

\section{Discussion}

To more accurately evaluate CRAI of a protease inhibitor and an antibiotic in patients with acute pancreatitis, we performed propensity score analysis based on largescale data from the Japanese nationwide administrative database. We found that CRAI was ineffective in that it did not reduce the in-hospital mortality rate, but rather was associated with an increased rate of interventions for infectious complications associated with acute pancreatitis. In addition, CRAI was associated with significantly longer hospital stays and significantly higher total costs.
Several small retrospective studies have suggested that CRAI may be effective in patients with severe acute pancreatitis [11-15]. To date, only one randomized controlled trial has shown that CRAI reduced mortality rates, but that study was limited by its relatively small sample size (78 patients) [16]. CRAI was originally performed in patients with pancreatic necrosis caused by acute pancreatitis, a condition with a high mortality rate. This treatment selection bias has hindered a simple retrospective comparison evaluating the efficacy of CRAI; that is, patients who underwent CRAI were likely to be at greater risk of acute pancreatitis-associated mortality, thus leading to underestimation of the efficacy of CRAI. The younger age of our CRAI cohort relative to our non-CRAI group was likely to enhance outcomes in the former [30], whereas the higher prognostic factor and CT severity index scores in the CRAI group would likely have a negative effect on outcomes [31]. Thus, to control for baseline prognostic heterogeneity and inherent treatment selection biases, we performed propensity score analysis [27,28]. We found that one-to-one propensity score matching resulted in a successful balance of baseline characteristics, including CT severity score, the factor that usually determines whether CRAI is administered.

When we compared the propensity-matched groups, we found that, in contrast to previous studies, in-hospital mortality rates were similar between our CRAI and nonCRAI groups, despite the tendency of earlier administration of CRAI to be associated with a lower in-hospital mortality rate. Although we expected that CRAI of an antibiotic would result in a lower rate of infectious complications, we found that the rate of requirement for 
Table 2 Characteristics of patients in the unmatched and propensity-matched groups with or without continuous regional arterial infusion ${ }^{\mathrm{a}}$

\begin{tabular}{|c|c|c|c|c|c|c|}
\hline \multirow[b]{2}{*}{ Patient characteristics } & \multicolumn{2}{|c|}{ Unmatched groups } & \multirow[b]{2}{*}{$P$} & \multicolumn{2}{|c|}{ Propensity-matched groups } & \multirow[b]{2}{*}{$P$} \\
\hline & CRAI $(n=247)$ & Non-CRAI $(n=1,307)$ & & CRAI $(n=207)$ & Non-CRAI $(n=207)$ & \\
\hline Mean age (years) & $54.6 \pm 16.4$ & $60.6 \pm 18.1$ & $<0.001$ & $56.7 \pm 16.3$ & $56.0 \pm 17.6$ & 0.684 \\
\hline \multicolumn{7}{|l|}{ Sex } \\
\hline Males & $69.2 \%$ & $67.6 \%$ & 0.622 & $69.6 \%$ & $66.7 \%$ & 0.527 \\
\hline Females & $30.8 \%$ & $32.4 \%$ & & $30.4 \%$ & $33.3 \%$ & \\
\hline \multicolumn{7}{|l|}{ CCl score } \\
\hline 0 & $47.4 \%$ & $43.1 \%$ & 0.404 & $48.8 \%$ & $48.3 \%$ & 0.944 \\
\hline 1 or 2 & $41.7 \%$ & $46.2 \%$ & & $42.5 \%$ & $42.0 \%$ & \\
\hline$\geq 3$ & $10.9 \%$ & $10.7 \%$ & & $8.7 \%$ & $9.7 \%$ & \\
\hline \multicolumn{7}{|l|}{ JCS score } \\
\hline 0 & $89.9 \%$ & $94.3 \%$ & 0.407 & $90.8 \%$ & $92.3 \%$ & 0.711 \\
\hline 1 to 3 & $6.9 \%$ & $4.5 \%$ & & $6.8 \%$ & $5.8 \%$ & \\
\hline 10 to 30 & $2.0 \%$ & $0.8 \%$ & & $1.4 \%$ & $0.5 \%$ & \\
\hline 100 to 300 & $1.2 \%$ & $0.4 \%$ & & $1.0 \%$ & $1.4 \%$ & \\
\hline \multicolumn{7}{|l|}{ Prognostic factor score } \\
\hline 0 & $29.1 \%$ & $48.3 \%$ & $<0.001$ & $34.3 \%$ & $35.3 \%$ & 0.970 \\
\hline 1 & $12.6 \%$ & $24.3 \%$ & & $14.0 \%$ & $14.5 \%$ & \\
\hline 2 & $17.4 \%$ & $11.1 \%$ & & $18.4 \%$ & $15.0 \%$ & \\
\hline 3 & $20.2 \%$ & $7.8 \%$ & & $16.9 \%$ & $16.9 \%$ & \\
\hline 4 & $12.1 \%$ & $3.6 \%$ & & $8.2 \%$ & $8.2 \%$ & \\
\hline 5 & $2.4 \%$ & $2.3 \%$ & & $2.4 \%$ & $2.4 \%$ & \\
\hline$\geq 6$ & $6.1 \%$ & $2.7 \%$ & & $5.8 \%$ & $7.7 \%$ & \\
\hline \multicolumn{7}{|l|}{ CT severity score } \\
\hline 0 & $13.4 \%$ & $47.8 \%$ & $<0.001$ & $15.9 \%$ & $15.5 \%$ & 0.999 \\
\hline 1 & $9.3 \%$ & $20.4 \%$ & & $11.1 \%$ & $10.6 \%$ & \\
\hline 2 & $38.9 \%$ & $21.7 \%$ & & $42.0 \%$ & $43.0 \%$ & \\
\hline 3 & $23.9 \%$ & $6.0 \%$ & & $18.4 \%$ & $18.8 \%$ & \\
\hline 4 & $14.6 \%$ & $4.1 \%$ & & $12.6 \%$ & $12.1 \%$ & \\
\hline \multicolumn{7}{|c|}{ Hospital volume (per 12 months) } \\
\hline$\leq 14$ & $21.5 \%$ & $20.0 \%$ & 0.324 & $21.7 \%$ & $24.6 \%$ & 0.835 \\
\hline 15 to 22 & $21.1 \%$ & $26.3 \%$ & & $22.2 \%$ & $23.7 \%$ & \\
\hline 23 to 32 & $24.3 \%$ & $24.3 \%$ & & $23.7 \%$ & $21.3 \%$ & \\
\hline$\geq 33$ & $33.2 \%$ & $29.4 \%$ & & $32.4 \%$ & $30.4 \%$ & \\
\hline \multicolumn{7}{|l|}{ Hospital type } \\
\hline Academic & $31.2 \%$ & $21.3 \%$ & 0.001 & $28.0 \%$ & $24.6 \%$ & 0.435 \\
\hline Community & $68.8 \%$ & $78.7 \%$ & & $72.0 \%$ & $75.4 \%$ & \\
\hline
\end{tabular}

${ }^{\mathrm{a} A g e}$ shown as mean and standard deviation. Prognostic factor score and CT severity score were based on the Japanese severity scoring system for acute pancreatitis (2008 revision) [22]. CCl, Charlson comorbidity index; CRAI, Continuous regional arterial infusion; CT, Computed tomography; JCS, Japan Coma Scale.

surgery and other interventions was higher in the CRAI group. In addition, hospital stay was significantly longer and total costs were significantly higher in the CRAI cohort than in the non-CRAI group.

This study has several limitations. First, it was not a prospective, randomized controlled trial, but rather was based on a retrospective design. Although we performed propensity score analysis to overcome this limitation, several biases may remain because of unobserved confounders. For example, the criteria for and timing of administration of CRAI were not predefined, and the changes in the prognostic factor and CT severity scores 
Table 3 Outcomes in the propensity-matched continuous regional arterial infusion and non-continuous regional arterial infusion groups ${ }^{\mathrm{a}}$

\begin{tabular}{lccc}
\hline Outcomes & CRAI $(\boldsymbol{n}=\mathbf{2 0 7})$ & Non-CRAI $(\boldsymbol{n}=\mathbf{2 0 7})$ & \multicolumn{1}{c}{$\boldsymbol{P}$} \\
\hline In-hospital mortality, $n(\%)$ & $16(7.7 \%)$ & $18.7 \%)$ & 0.720 \\
Median length of stay, days (IQR) & $28.5(18.3$ to 36.8$)$ & $(12.0$ to 28.0$)$ & $<0.001$ \\
Median cost, US\$ (IQR) & $\$ 21,800(\$ 16,200$ to $\$ 32,400)$ & $12,600(\$ 7,940$ to $\$ 21,700)$ & $<0.001$ \\
Interventions for infectious complications, $n(\%)$ & $6(2.9 \%)$ & $1(0.5 \%)$ & 0.061 \\
\hline
\end{tabular}

${ }^{a} \mathrm{CRAl}$, Continuous regional arterial infusion; IQR, Interquartile range.

over time were unavailable from the DPC database. Thus, the condition of some patients diagnosed with "nonsevere" acute pancreatitis upon admission may have worsened rapidly, thus unfavorably affecting the outcomes in our CRAI group. Second, CT severity score was determined by scoring and summing two independent factors: extrapancreatic progression of inflammation and hypoenhanced lesions of the pancreas. These two factors affect the efficacy of CRAI differently, although total CT severity scores were well-balanced in our adjusted CRAI and non-CRAI groups. Third, the unavailability of some important clinical data from the DPC database may have affected patient outcomes, including the etiologies of acute pancreatitis [32,33], symptoms of sepsis, data on organ failure, dosages of protease inhibitors and antibiotics on a daily basis and detailed results of blood tests and CT. The information on readmissions and outpatient visits was also unavailable, which inhibited the analysis of late mortality associated with acute pancreatitis. The multiplicity of protease inhibitors and antibiotics used constitutes another limitation.

Despite these limitations, a major strength of this study was the evaluation of CRAI for acute pancreatitis in a large number of patients with well-balanced baseline characteristics, including prognostic factor score affecting inhospital mortality rate, such as age [30], sex [32,34], consciousness [30], prognostic factor score [31] and CT severity score. To the best of our knowledge, this study is the largest to date that has evaluated CRAI, and it included patients who underwent CRAI in a large number of hospitals $(n=99)$, including 70 nonacademic hospitals with a relatively low hospital volume and 29 academic hospitals, thus providing internal validity. Because of its invasiveness and cost, CRAI should not routinely be performed in patients with acute pancreatitis until its efficacy has been well-established and its indications are confirmed.

\section{Conclusions}

On the basis of our propensity score analysis of largescale data from a nationwide administrative database, we found that CRAI was not effective in the treatment of acute pancreatitis and that it cost more. Well-designed, randomized controlled trials including large numbers of patients are needed to further evaluate the efficacy of this procedure.

\section{Key messages}

- Because little is known about the potential effectiveness of CRAI in patients with severe acute pancreatitis, the effectiveness and costs of CRAI were evaluated on the basis of data derived from a nationwide, large-scale database.

- One-to-one propensity score matching was performed to adjust for treatment selection bias, and 207 well-balanced pairs were compared.

- CRAI failed to demonstrate superiority regarding mortality rate, length of hospital stay and total cost, suggesting the need for large randomized controlled trials to further evaluate the effectiveness of CRAI.

Table 4 Odds ratios for in-hospital mortality and interventions for infectious complications and coefficients for length of stay and cost of the CRAI group, compared with the non-CRAI group

\begin{tabular}{|c|c|c|c|c|}
\hline \multirow[b]{2}{*}{ Variable } & \multicolumn{3}{|c|}{ CRAI vs. non-CRAI } & \multirow[b]{2}{*}{$P$} \\
\hline & Odds ratio & Coefficient & $95 \% \mathrm{Cl}$ & \\
\hline In-hospital mortality & 0.88 & & 0.43 to 1.78 & 0.711 \\
\hline Interventions for infectious complications & 6.42 & & 0.75 to 54.6 & 0.089 \\
\hline Length of stay (days) & & 16.5 & 11.8 to 21.2 & $<0.001$ \\
\hline Cost (US dollars) & & $\$ 13,600$ & 6,890 to 20,400 & $<0.001$ \\
\hline
\end{tabular}

The odds ratios were calculated using logistic regression analysis with adjustment for propensity score quintiles, and the coefficients using linear regression analysis with adjustment for age, sex, JCS, CCl, prognostic factor score, CT score, hospital volume and hospital type. 


\section{Abbreviations}

CCl: Charlson comorbidity index; Cl: Confidence interval; CRAl: Continuous regional arterial infusion; CT: Computed tomography; DPC: Diagnosis procedure combination; ICD-10: International classification of diseases and related health problems, 10th revision; IQR: Interquartile range; JCS: Japan coma scale; OR: Odds ratio.

\section{Competing interests}

The authors declare that they have no competing interest.

\section{Authors' contributions}

TH participated in the study concept and design, analysis and interpretation of data, drafting of the manuscript and statistical analysis. HY was responsible for acquisition of data, study concept and design, analysis and interpretation of data, statistical analysis and critical revision of the manuscript for important intellectual content. YN and HI participated in the study concept and design, analysis and interpretation of data and critical revision of the manuscript for important intellectual content. HH, SM and KF were involved with acquisition of data and critical revision of the manuscript for important intellectual content. KK was responsible for study supervision and final approval of the manuscript. All authors read and approve the final manuscript.

\section{Acknowledgements}

This study was funded by a Grant-in-Aid for Research on Policy Planning and Evaluation from the Ministry of Health, Labour and Welfare, Japan (H22-Policy-031), by a Grant-in-Aid for Scientific Research B (22390131) from the Ministry of Education, Culture, Sports, Science and Technology and by the Funding Program for World-Leading Innovative R\&D on Science and Technology (FIRST program) from the Council for Science and Technology Policy, Japan (0301002001001)

\section{Author details}

'Department of Gastroenterology, Graduate School of Medicine, The University of Tokyo, 7-3-1 Hongo, Bunkyo-ku, Tokyo 113-8655, Japan. ${ }^{2}$ Department of Health Economics and Epidemiology Research, School of Public Health, The University of Tokyo, 7-3-1 Hongo, Bunkyo-ku, Tokyo 113-8655, Japan. ${ }^{3}$ Department of Clinical Data Management and Research, Clinical Research Center, National Hospital Organization Headquarters, 2-5-21 Higashigaoka, Meguro-ku, Tokyo 152-8621, Japan. ${ }^{4}$ Department of Preventive Medicine and Community Health, University of Occupational and Environmental Health, 1-1 Iseigaoka, Yahatanishi-ku, Kitakyushu 807-8555, Japan. ${ }^{5}$ Department of Health Care Informatics, Tokyo Medical and Dental University, 1-5-45 Yushima, Bunkyo-ku, Tokyo 113-8510, Japan.

Received: 25 May 2013 Accepted: 26 July 2013

Published: 2 October 2013

\section{References}

1. Kingsnorth A, O'Reilly D: Acute pancreatitis. BMJ 2006, 332:1072-1076.

2. Frossard JL, Steer ML, Pastor CM: Acute pancreatitis. Lancet 2008, 371:143-152.

3. Nathens AB, Curtis JR, Beale RJ, Cook DJ, Moreno RP, Romand JA, Skerrett SJ, Stapleton RD, Ware LB, Waldmann CS: Management of the critically ill patient with severe acute pancreatitis. Crit Care Med 2004, 32:2524-2536.

4. Sekimoto M, Takada T, Kawarada Y, Hirata K, Mayumi T, Yoshida M, Hirota M, Kimura Y, Takeda K, Isaji S, Koizumi M, Otsuki M, Matsuno S, JPN: JPN Guidelines for the management of acute pancreatitis: epidemiology, etiology, natural history, and outcome predictors in acute pancreatitis. J Hepatobiliary Pancreat Surg 2006, 13:10-24.

5. Chen HM, Chen JC, Hwang TL, Jan YY, Chen MF: Prospective and randomized study of gabexate mesilate for the treatment of severe acute pancreatitis with organ dysfunction. Hepatogastroenterology 2000, 47:1147-1150.

6. Seta T, Noguchi Y, Shimada T, Shikata S, Fukui T: Treatment of acute pancreatitis with protease inhibitors: a meta-analysis. Eur J Gastroenterol Hepatol 2004, 16:1287-1293.

7. Sainio V, Kemppainen E, Puolakkainen P, Taavitsainen M, Kivisaari L, Valtonen V, Haapiainen R, Schröder T, Kivilaakso E: Early antibiotic treatment in acute necrotising pancreatitis. Lancet 1995, 346:663-667.

8. Golub R, Siddiqi F, Pohl D: Role of antibiotics in acute pancreatitis: a meta-analysis. J Gastrointest Surg 1998, 2:496-503.
9. Yamauchi J, Takeda K, Shibuya K, Sunamura M, Matsuno S: Continuous regional application of protease inhibitor in the treatment of acute pancreatitis: an experimental study using closed duodenal obstruction model in dogs. Pancreatology 2001, 1:662-667.

10. Mikami Y, Takeda K, Matsuda K, Qiu-Feng H, Fukuyama S, Egawa S, Sunamura M, Matsuno S: Rat experimental model of continuous regional arterial infusion of protease inhibitor and its effects on severe acute pancreatitis. Pancreas 2005, 30:248-253.

11. Takeda K, Matsuno S, Sunamura M, Kakugawa Y: Continuous regional arterial infusion of protease inhibitor and antibiotics in acute necrotizing pancreatitis. Am J Surg 1996, 171:394-398.

12. Takeda K, Matsuno S, Ogawa M, Watanabe S, Atomi Y: Continuous regional arterial infusion (CRAI) therapy reduces the mortality rate of acute necrotizing pancreatitis: results of a cooperative survey in Japan. $J$ Hepatobiliary Pancreat Surg 2001, 8:216-220.

13. Imaizumi H, Kida M, Nishimaki H, Okuno J, Kataoka Y, Kida Y, Soma K, Saigenji K: Efficacy of continuous regional arterial infusion of a protease inhibitor and antibiotic for severe acute pancreatitis in patients admitted to an intensive care unit. Pancreas 2004, 28:369-373.

14. Takeda K: Antiproteases in the treatment of acute necrotizing pancreatitis: continuous regional arterial infusion. JOP 2007, 8:526-532.

15. Ino $Y$, Arita Y, Akashi T, Kimura T, Igarashi H, Oono T, Furukawa M, Kawabe K, Ogoshi K, Ouchi J, Miyahara T, Takayanagi R, Ito T: Continuous regional arterial infusion therapy with gabexate mesilate for severe acute pancreatitis. World J Gastroenterol 2008, 14:6382-6387.

16. Piascik M, Rydzewska G, Milewski J, Olszewski S, Furmanek M, Walecki J, Gabryelewicz A: The results of severe acute pancreatitis treatment with continuous regional arterial infusion of protease inhibitor and antibiotic: a randomized controlled study. Pancreas 2010, 39:863-867.

17. Shanbhag ST, Petrov MS, Windsor JA: Is continuous regional arterial infusion of antiproteases now a standard of care in the treatment of acute pancreatitis? Pancreas 2011, 40:1141.

18. Yasunaga $H$, Hashimoto $H$, Horiguchi $H$, Miyata $H$, Matsuda S: Variation in cancer surgical outcomes associated with physician and nurse staffing: a retrospective observational study using the Japanese Diagnosis Procedure Combination Database. BMC Health Serv Res 2012, 12:129.

19. Yasunaga $H$, Horiguchi $H$, Matsuda $S$, Fushimi $K$, Hashimoto $H$, Ohe $K$, Kokudo N: Relationship between hospital volume and operative mortality for liver resection: data from the Japanese diagnosis procedure combination database. Hepatol Res 2012, 42:1073-1080.

20. Todo T, Usui M, Takakura $K$ : Treatment of severe intraventricular hemorrhage by intraventricular infusion of urokinase. J Neurosurg 1991, 74:81-86.

21. Ono K, Wada K, Takahara T, Shirotani T: Indications for computed tomography in patients with mild head injury. Neurol Med Chir (Tokyo) 2007, 47:291-298.

22. Takeda K, Yokoe M, Takada T, Kataoka K, Yoshida M, Gabata T, Hirota M, Mayumi T, Kadoya M, Yamanouchi E, Hattori T, Sekimoto M, Amano H, Wada K, Kimura Y, Kiriyama S, Arata S, Takeyama Y, Hirota M, Hirata K, Shimosegawa T: Assessment of severity of acute pancreatitis according to new prognostic factors and CT grading. J Hepatobiliary Pancreat Sci 2010, 17:37-44.

23. Mounzer R, Langmead CJ, Wu BU, Evans AC, Bishehsari F, Muddana V, Singh VK, Slivka A, Whitcomb DC, Yadav D, Banks PA, Papachristou GI: Comparison of existing clinical scoring systems to predict persistent organ failure in patients with acute pancreatitis. Gastroenterology 2012, 142:1476-1482. quiz e15-e16.

24. Hamada T, Yasunaga H, Nakai Y, Isayama H, Horiguchi H, Fushimi K, Koike K: Japanese severity score for acute pancreatitis well predicts in-hospital mortality: a nationwide survey of 17,901 cases. $J$ Gastroenterol. in press. doi:10.1007/s00535-013-0765-6.

25. Quan H, Sundararajan V, Halfon P, Fong A, Burnand B, Luthi JC, Saunders LD, Beck CA, Feasby TE, Ghali WA: Coding algorithms for defining comorbidities in ICD-9-CM and ICD-10 administrative data. Med Care 2005, 43:1130-1139.

26. Singla A, Simons J, Li Y, Csikesz NG, Ng SC, Tseng JF, Shah SA: Admission volume determines outcome for patients with acute pancreatitis. Gastroenterology 2009, 137:1995-2001.

27. Joffe MM, Rosenbaum PR: Invited commentary: propensity scores. Am J Epidemiol 1999, 150:327-333.

28. D'Agostino RB Jr: Propensity score methods for bias reduction in the comparison of a treatment to a non-randomized control group. Stat Med 1998, 17:2265-2281. 
29. Rosenbaum PR, Rubin DB: Constructing a control-group using multivariate matched sampling methods that incorporate the propensity score. Am Stat 1985, 39:33-38.

30. Wu BU, Johannes RS, Sun X, Tabak Y, Conwell DL, Banks PA: The early prediction of mortality in acute pancreatitis: a large population-based study. Gut 2008, 57:1698-1703.

31. Ueda T, Takeyama Y, Yasuda T, Kamei K, Satoi S, Sawa H, Shinzeki M, Ku Y, Kuroda $Y$, Ohyanagi $H$ : Utility of the new Japanese severity score and indications for special therapies in acute pancreatitis. J Gastroenterol 2009, 44:453-459.

32. Pezzilli R, Billi P, Morselli-Labate AM: Severity of acute pancreatitis: relationship with etiology, sex and age. Hepatogastroenterology 1998, 45:1859-1864.

33. Lankisch PG, Assmus C, Pflichthofer D, Struckmann K, Lehnick D: Which etiology causes the most severe acute pancreatitis? Int J Pancreatol 1999, 26:55-57.

34. Spanier BW, Dijkgraaf MG, Bruno MJ: Epidemiology, aetiology and outcome of acute and chronic pancreatitis: an update. Best Pract Res Clin Gastroenterol 2008, 22:45-63.

doi:10.1186/cc13029

Cite this article as: Hamada et al:: Continuous regional arterial infusion

for acute pancreatitis: a propensity score analysis using a nationwide administrative database. Critical Care 2013 17:R214.

\section{Submit your next manuscript to BioMed Central and take full advantage of:}

- Convenient online submission

- Thorough peer review

- No space constraints or color figure charges

- Immediate publication on acceptance

- Inclusion in PubMed, CAS, Scopus and Google Scholar

- Research which is freely available for redistribution 